\title{
Lutzomyia umbratilis from an area south of the Negro River is refractory to in vitro interaction with Leishmania guyanensis
}

\author{
${ }^{1}$ Fundação Oswaldo Cruz-Fiocruz, Instituto René Rachou, Belo Horizonte, MG, Brasil \\ ${ }^{2}$ Fundação Oswaldo Cruz-Fiocruz, Instituto Leônidas e Maria Deane, Manaus, AM, Brasil
}

Rodrigo Pedro Soares ${ }^{1}$, Paula Monalisa Nogueira1', Nágila Francinete Secundino', Eric Fabrício Marialva², Cláudia Maria Ríos-Velásquez ${ }^{2}$, Felipe Arley Costa Pessoa ${ }^{2}{ }^{+}$

BACKGROUND Lutzomyia umbratilis, the vector for Leishmania guyanensis in northern South America, has been found naturally infected with L. guyanensis only in areas north of the Negro and Amazon rivers. While populations of this sand fly species are also found in areas south of these rivers, these populations have never been reported to be infected and/or transmitting $L$. guyanensis. However, no studies on the corresponding host-parasite interactions are available.

OBJECTIVES This study evaluated the interaction between $L$. guyanensis promastigotes and field-collected Lu. umbratilis sand flies from Rio Preto da Eva and Manacapuru, which are located to the north and south, respectively, of the Negro River.

METHODS Procyclic and metacyclic attachment was quantified using an in vitro system.

FINDINGS Low attachment of parasites to the midguts of insects collected from Manacapuru was detected. Conversely, greater binding of metacyclic parasites was observed in the midguts of insects collected from Rio Preto da Eva, and this attachment was more pronounced than that observed for procyclics $(\mathrm{p}<0.03)$.

MAIN CONCLUSIONS The Lu. umbratilis population from an area south of the Negro River has lower in vitro interaction with L. guyanensis. The higher attachment of L. guyanensis to midguts of insects from Rio Preto da Eva may suggest better vector competence. These findings are in accordance with previously reported epidemiological information of American cutaneous leishmaniasis (ACL) transmission in the Amazon.

Key words: Lutzomyia umbratilis - Leishmania guyanensis - host-parasite interaction - American cutaneous leishmaniasis (ACL)

The sand fly Lutzomyia (Nyssomyia) umbratilis Ward \& Frahia is the main vector of Leishmania (Viannia) guyanensis, an infectious agent of American cutaneous leishmaniasis (ACL) in Northern South America (Ready et al. 1985). In addition to its distribution in the Amazonian basin (Young \& Duncan 1994), this species has also been detected in Atlantic Rainforest remnants in Northeast Brazil (Balbino et al. 2001); however, its local role as a transmission agent of ACL in Northeast Brazil is yet to be determined (Freitas et al. 2017).

Lu. umbratilis has been found to be naturally infected with L. guyanensis only in areas east or north of the Negro River (Arias \& Freitas 1977, 1978). Arias and Freitas (1978) suggested that this river could act as a vicariant barrier to L. guyanensis transmission. Justiniano et al. (2004) compared the first laboratory bred generations of Lu. umbratilis populations obtained from areas south and north of the Negro River. Those populations exhibited remarkable biological differences in their life cycle, fecundity, fertility, and adult longevity. The northern population was more productive and lived longer compared to the southern population. These differences could be due

doi: 10.1590/0074-02760170425

Financial support: CNPq, FAPEMIC

+ Corresponding author: felipe.pessoa@fiocruz.br

Received 08 October 2017

Accepted 28 November 2017 to intrinsic biological features resulting from their geographical isolation by the Negro River. Later, Scarpassa and Alencar (2012) compared Lu. umbratilis populations from Manacapuru (Man; south of the Negro River) and Rio Preto da Eva (RPE; north of the Negro River) by using the cytochrome oxidase I (COI) gene and suggested that, based on its clustering, the Man population could be undergoing speciation. More recently, Freitas et al. $(2015,2016)$ compared these populations and included Lu. umbratilis specimens from Recife (in Northeast Brazil). Consistent with previous observations, two distinct clades developed: one including Lu. umbratilis populations from RPE and Recife and another including only the Lu. umbratilis population from Man.

A distinguishing feature in the epidemiology of ACL in the Manaus region is the complete absence of L. guyanensis transmission by Lu. umbratilis in Man, a city located south of the Negro River, where neither infected insects were captured nor were ACL patients found. On the other hand, in RPE, which is located north of the Negro River, cases of infection in humans often occur and infected insects are found. To understand if these distinct Lu. umbratilis populations have different abilities to interact with L. guyanensis, in vitro binding experiments were performed (Pimenta et al. 1992). This system allows a rapid quantitative analysis of the interaction between insect midguts and promastigote forms of Leishmania.

Cell culture - The World Health Organization reference strain of L. guyanensis (MHOM/BR/75/M4147) was used. This strain was isolated by Dr Ralph Lainson 
from a human case in Monte Dourado, northern Pará state, Brazil (Lainson et al. 1981). Promastigotes (unknown passage) were cultured in M199 medium supplemented with $10 \%$ heat inactivated foetal bovine serum (FBS), 100 units $/ \mathrm{mL}$ penicillin, $50 \mu \mathrm{g} / \mathrm{mL}$ streptomycin, $12.5 \mathrm{mM}$ glutamine, $0.1 \mathrm{M}$ adenine, $0.0005 \%$ hemin, and $40 \mathrm{mM}$ HEPES, $\mathrm{pH} 7.4$, at $26^{\circ} \mathrm{C}$ until they reached stationary phase (Soares et al. 2002).

Purification of peanut agglutinin (PNA)-negative cells - Parasites from the stationary phase $\left(1.0 \times 10^{7}\right.$ cells $/ \mathrm{mL}$ ) were harvested and resuspended in M199 containing PNA (Arachis hypogaea) at a final concentration of $35 \mu \mathrm{g} / \mathrm{mL}$. This lectin has been successfully used for purification of metacyclic promastigotes in this species (Muskus et al. 1997). After a 30-min incubation at room temperature, the agglutinated parasites $(\mathrm{PNA}+)$ were removed by low-speed centrifugation $(150 \times g, 5 \mathrm{~min})$, and metacyclic cells remaining in the supernatant (PNA) were washed twice with phosphate-buffered saline (PBS) at $2100 \times g$ for $15 \mathrm{~min}$ at $4^{\circ} \mathrm{C}$ (Soares et al. 2005).

Midgut binding studies - Field-collected Lu. umbratilis sand flies from RPE (north of the Negro River, Amazonas; $2^{\circ} 50^{\prime} 50^{\prime \prime} \mathrm{S} / 59^{\circ} 56^{\prime} 28^{\prime \prime} \mathrm{W}$ ) and Man (south of the Negro River; $3^{\circ} 12^{\prime} 41^{\prime \prime} \mathrm{S} / 60^{\circ} 26^{\prime} 20$ ) were used in the experiments. Both municipalities are in the northern Amazonas state, Brazil (Fig. 1). Insects were collected from tree trunk crevices by using CDC traps between 10:00 am and 12:00 pm. Taxonomic identification was performed prior to dissecting the midguts according to Young and Duncan (1994). Two consecutive binding experiments (July 2015 and August 2015) were performed in the Laboratory of Transmissible Diseases Ecology in the Amazon (ILMD/FIOCRUZ) in Manaus, Amazonas state, Brazil. In the first experiment (July 2015), 11 insects per group (PNA+ and PNA-) were dissected for each locality (Man and RPE), totaling 44 insects. In the second experiment (August 2015), 11 insects per group (PNA+ and PNA-) were dissected for each locality (Man and RPE), totaling 44 insects. Promastigote binding was quantified by an in vitro technique (Pimenta et al. 1994). Blood-unfed females maintained on $30 \%$ sucrose were dissected in PBS. Midguts were opened along the length of the abdominal segment by using a fine needle, placed in the concave wells of a microscope chamber slide, and individually incubated for $20 \mathrm{~min}$ with procyclic (PNA+) and metacyclic (PNA-) promastigotes $\left(2 \times 10^{7}\right.$ cells $/ \mathrm{mL}$ in a volume of $50 \mu \mathrm{L}$ ). Then, guts were washed in successive drops of PBS, and the number of attached parasites was determined using a Neubauer-counting chamber.

Data analysis - The Shapiro-Wilk test was employed to test the null hypothesis that data were sampled from a Gaussian distribution, where $p$ values $<0.05$ would indicate deviation from a Gaussian distribution. In such cases, a non-parametric Kruskal-Wallis test was performed, followed by Dunn's test. The Mann-Whitney test was used to compare both experiments (July and August 2015); as there were no significant differences between the two experiments ( $p>0.05$; Table), data were grouped (Fig. 2) and analysed using GraphPad Prism version 5.00 for Windows. (GraphPad Software, La Jolla, CA, USA; www.graphpad. com). $\mathrm{P}<0.05$ was considered statistically significant.

Attachment to the sand fly midgut is a crucial step for Leishmania survival in the vector, and this is one of the interactions that determines vector competence. In Old World species such as Phlebotomus papatasi and Leishmania major, binding to the PpGalec receptor is mediated by lipophosphoglycan (LPG), a major Leishmania surface glycoconjugate (Kamhawi et al. 2004). However, a receptor for parasite attachment in New World species of sand flies is yet to be determined, although a midgut mucin in Lu. longipalpis was suggested (Myskova et al. 2016).

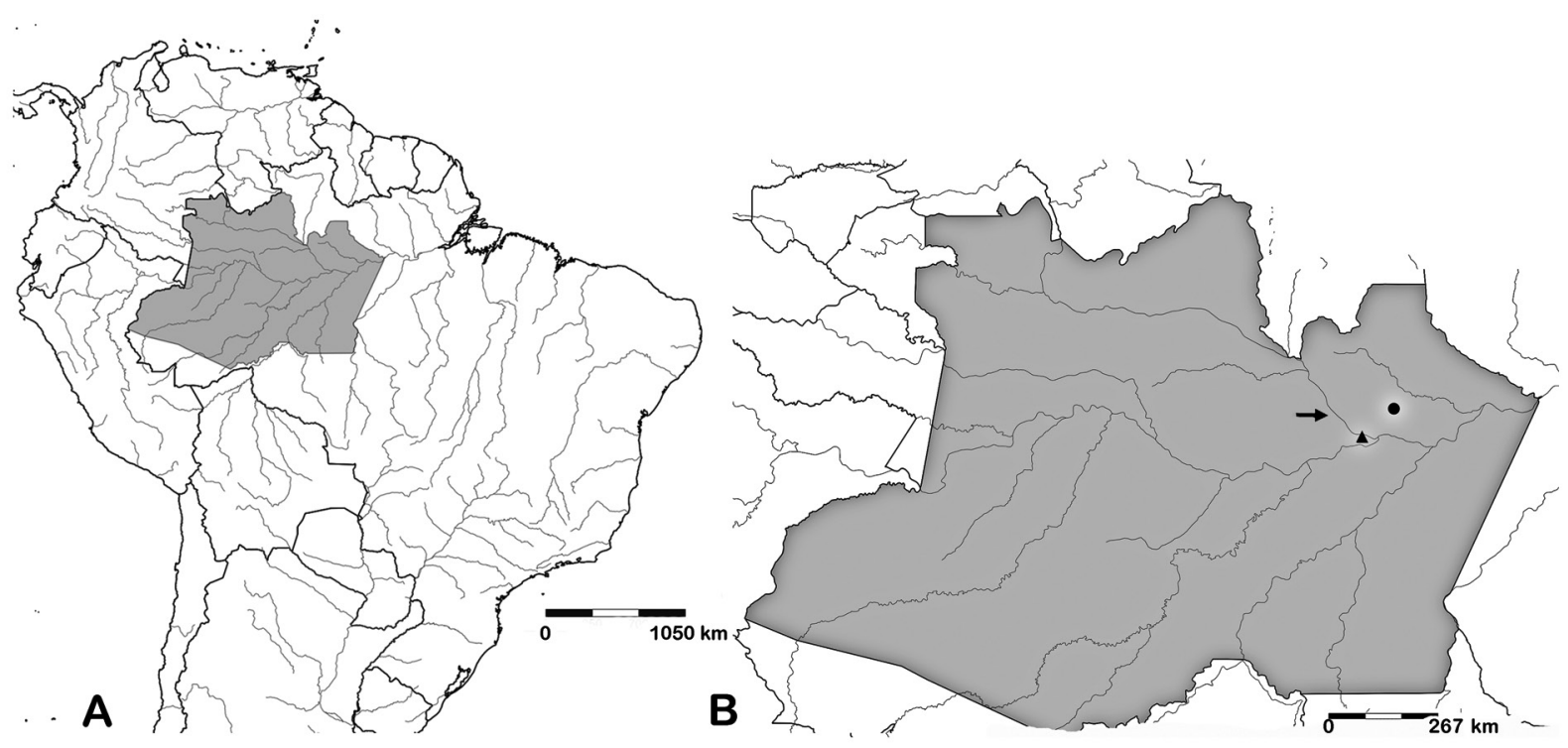

Fig. 1: geographic location of Lutzomyia umbratilis populations. (A) Map of South America showing the Amazonas state, Brazil (grey); (B) collection areas in Amazonas: Rio Preto da Eva (dark circle) and Manacapuru (dark triangle). The Negro River as geographical barrier (arrow). 
TABLE

In vitro attachment of Leishmania guyanensis to the midgut of Lutzomyia umbratilis collected from two different locations

\begin{tabular}{|c|c|c|c|}
\hline & July 2015 & August 2015 & \\
\hline & median (Q1, Q3) & median (Q1, Q3) & $\mathrm{p}$ value \\
\hline Man PNA+ x Man PNA+ & $0.00(0.00,0.00)$ & $0.00(0.00,0.00)$ & $>0.99$ \\
\hline Man PNA- x Man PNA- & $0.00(0.00,0.00)$ & $0.00(0.00,0.00)$ & $>0.99$ \\
\hline RPE PNA+ $x$ RPE PNA+ & $5,195.00(2995.00,6,593.00)$ & 2,398.00 (1,399.00, 2,997.00) & 0.08 \\
\hline RPE PNA- $x$ RPE PNA- & $1,798.00(599.40,4,795.00)$ & $24,726.00(1,199.00,44,356.00)$ & 0.30 \\
\hline
\end{tabular}

RPE: Rio Preto da Eva; Man: Manacapuru; PNA+: procyclic; PNA-: metacyclic; Q1: 25th percentile; Q3: (75th percentile). p $<0.05$ was considered statistically significant.

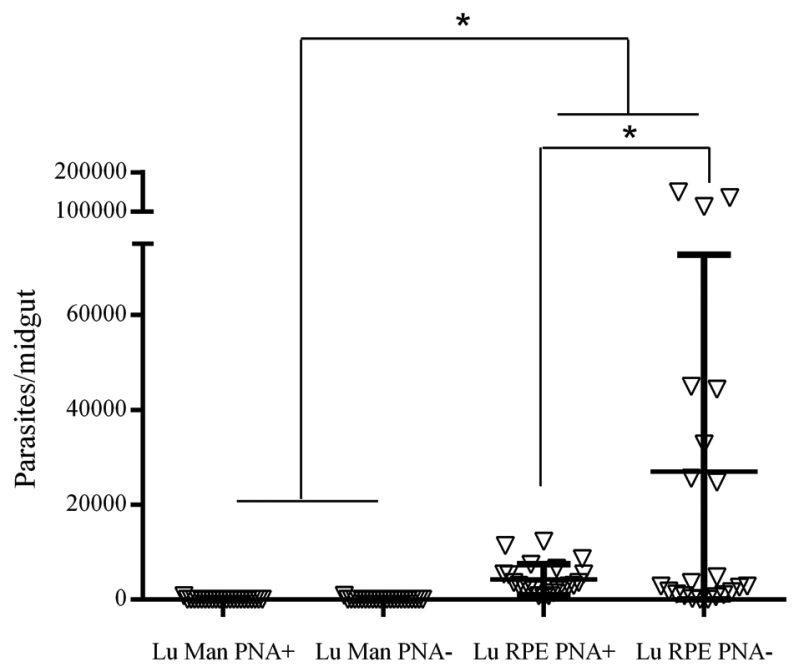

Fig. 2: attachment of procyclic (PNA+) and metacyclic (PNA-) Leishmania guyanensis promastigotes to Lutzomyia umbratilis (Lu) midguts. Man: Manacapuru; RPE: Rio Preto da Eva. Data represents two independent experiments. Asterisks $\left(^{*}\right)$ indicate statistically significant differences $(\mathrm{p}<0.03)$.

In vitro and in vivo protocols are available to determine parasite binding and survival inside the vector, respectively. In vitro protocols were developed in the early 90 s to evaluate the role of phosphoglycans (PGs) in the Old World vectors Ph. papatasi and Phlebotomus argentipes (Pimenta et al. 1992, 1994). Later, the in vitro system was expanded to the New World species/strains $L$. infantum and Lu. longipalpis (Soares et al. 2002, 2017); L. braziliensis, Lu. whitmani, and Lu. intermedia (Soares et al. 2010, 2017); and L. amazonensis, Lu. flaviscutellata, Lu. longipalpis, and Lu. antunesi (de Oliveira et al. 2016).

Here, we used this in vitro protocol to determine the vector-parasite interaction in two Lu. umbratilis populations in endemic (RPE, AM) and non-endemic areas (Man, AM) for ACL. The in vitro protocol was selected for this study because, under the current conditions, $L u$. umbratilis is not colonised to provide a productive offspring (Justiniano et al. 2004). Besides, females captured from the wild refuse to feed using the artificial feeding device (FA Pessoa, unpublished data, ILMD/FIOCRUZ).
After incubation of procyclic (PNA + ) and metacyclic (PNA-) L. guyanensis promastigotes with RPE and AM sand fly populations, binding to the midgut was higher in insects from RPE in both experiments $(p<0.01)$. Low parasite attachment was observed in the midguts of $L u$. umbratilis captured in Man. A unique feature of Lu. umbratilis binding was observed in RPE sandflies where metacyclic parasites bound to the midguts 11-fold more often than the procyclic ones (Table, Fig. 2). L. guyanensis is a perypilarian species from the subgenus Viannia, which develops in the pyloric triangle before migration to the midgut and foregut (Lainson \& Shaw 1987). Previous studies showed that procyclic and metacyclic promastigotes from L. braziliensis, another Viannia subgenus species, could bind to the midgut of Lu. whitmani and Lu. intermedia (Soares et al. 2010). This is different from the subgenus Leishmania, where only procyclic parasites and/or PGs successfully bind to the epithelium (Pimenta et al. 1994, Mahoney et al. 1999, Kamhawi et al. 2000, Soares et al. 2002). The ability of L. braziliensis promastigotes to bind to the sand fly midgut may vary depending on the vector. Procyclic bind more than metacyclic promastigotes to the $\mathrm{Lu}$. whitmani midgut, whereas in Lu. intermedia, they bound equally (Soares et al. 2010). It was hypothesized, based on higher attachment of $L$. braziliensis PGs, that in the species of the subgenus $\mathrm{Vi}$ annia, a "second metacyclic stage" could be responsible for detachment from the midgut and further migration to the foregut (Kamhawi 2006, Assis et al. 2012). Similar to the observations in L. braziliensis metacyclic promastigotes, this is the first demonstration that L. guyanensis metacyclic promastigotes could bind to Lu. umbratilis midguts. However, the demonstration of a "second metacyclic stage" in this species is yet to be determined using in vivo experiments. Although the in vitro system has limitations (Wilson et al. 2010), it was proven to be very successful for studies on natural vector-parasite pairs.

The complete lack of interaction between L. guyanensis and the midgut surface of Lu. umbratilis collected from Man may reflect the genetic and morphometric differences in this population (Scarpassa \& Alencar 2012, Freitas et al. 2015, 2016).

In conclusion, the findings herein provide the first evidence that Lu. umbratilis from south of the Negro River 
is extremely refractory to interaction with L. guyanensis, which supports previous epidemiological data indicating no ACL transmission due to this species in this area. According to the Killick-Kendrick (1999), Lu. umbratilis is a proven vector of $L$. guyanensis because it fulfills all five requirements for vectorial capacity. Furthermore, its vectorial capacity relies on many ecological factors in endemic areas corresponding to not only to their natural infection but also to their ability to transmit the parasite to humans (Lainson et al. 1981). However, the lack of in vitro interaction between L. guyanensis and insects from the Man population captured in non-endemic areas may suggest low vector competence of these insects, leading to lower vectorial capacity. Conversely, under the current experimental conditions of the in vitro system, specimens from the northern population (those from RPE) exhibit higher vector competence, which may reflect their improved vectorial ability for transmitting ACL in RPE.

\section{REFERENCES}

Arias JR, Freitas RA. On the vectors of cutaneous leishmaniasis in the central Amazon of Brazil. I. Preliminary findings. Acta Amazonica. 1977; 7(2): 293-4.

Arias JR, Freitas RA. Sobre os vetores da leishmaniose cutânea na Amazônia Central do Brasil. 2. Incidência de flagelados em flebótomos selváticos. Acta Amazonica. 1978; 8(3): 387-96.

Assis RR, Ibraim IC, Nogueira PM, Soares RP, Turco SJ. Glycoconjugates in New World species of Leishmania: polymorphisms in lipophosphoglycan and glycoinositolphospholipids and interaction with hosts. Bioch Bioph Acta General Subjects. 2012; 1820(9): 1354-65.

Balbino VQ, Marcondes CB, Alexander B, Luna LKS, Lucena MMM, Mendes ACS, et al. First report of Lutzomyia (Nyssomyia) umbratilis Ward \& Frahia, 1977 outside of Amazonian Region, in Recife, state of Pernambuco, Brazil (Diptera: Psychodidae: Phlebotominae). Mem Inst Oswaldo Cruz. 2001; 96(3): 315-7.

de Oliveira DM, da Silva BJ, de Sena CB, Lima JA, dos Santos TV, Silveira FT, et al. Comparative analysis of carbohydrate residues in the midgut of phlebotomines (Diptera: Psychodidae) from colony and field populations from Amazon, Brazil. Exp Parasitol. 2016; 168: 31-8.

Freitas MTS, Ríos-Velásquez CM, Costa Jr CR, Figueirêdo Jr CA, Aragão NC, da Silva LG, et al. Phenotypic and genotypic variations among three allopatric populations of Lutzomyia umbratilis, main vector of Leishmania guyanensis. Parasit Vectors. 2015; 8: 448.

Freitas MTS, Ríos-Velásquez CM, da Silva LG, Costa Jr CR, Marcelino A, Leal-Balbino TC, et al. Analysis of the genetic structure of allopatric populations of Lutzomyia umbratilis using the period clock gene. Acta Trop. 2016; 154: 149-54.

Freitas MTS, Santos FR, Andrade EM, Marcondes CB, Balbino VQ, Pessoa FA. New records of Phlebotomine sand flies (Diptera: Psychodidae) from the state of Alagoas, Northeast of Brazil. J Med Entomol. 2017; doi: 10.1093/jme/tjx175.

Justiniano SC, Chagas AC, Pessoa FA, Queiroz RG. Comparative biology of two populations of Lutzomyia umbratilis (Diptera: Psychodidae) of Central Amazonia, Brazil, under laboratory conditions. Braz J Biol. 2004; 64(2): 227-35.

Kamhawi S, Modi GB, Pimenta PFP, Rowton E, Sacks DL. The vectorial competence of Phlebotomus sergenti is specific for Leishmania tropica and is controlled by species-specific, lipophosphoglycanmediated midgut attachment. Parasitology. 2000; 121(Pt 1): 25-33.

Kamhawi S, Ramalho-Ortigão M, Pham VM, Kumar S, Lawyer PG, Turco SJ, et al. Role for insect galectins in parasite survival. Cell. 2004; 119(3): 329-41.
Kamhawi S. Phlebotomine sand flies and Leishmania parasites: friends or foes? Trends Parasitol. 2006; 22(9): 439-45.

Killick-Kendrick R. The biology and control of phlebotomine sand flies. Clin Dermatol. 1999; 17(3): 279-89.

Lainson R, Shaw JJ, Ready PD, Miles MA, Póvoa M. Leishmaniasis in Brazil: XVI. Isolation and identification of Leishmania species from sandflies, wild mammals and man in north Para state, with particular reference to L. braziliensis guyanensis causative agent of “pian-bois”. Trans R Soc Trop Med Hyg. 1981; 75(4): 530-6.

Lainson R, Shaw JJ. Evolution, classification and geographical distribution. In: Peters W, Killick-Kendrick R, editors. The leishmaniasis in biology and medicine. Vol. I. London: Academic Press; 1987. 120 pp.

Mahoney AB, Sacks DL, Saraiva E, Modi G, Turco SJ. Intra-species and stage-specific polymorphisms in lipophosphoglycan structure control Leishmania donovani-sand fly interactions. Biochemistry. 1999; 38(31): 9813-23.

Muskus C, Segura I, Odone R, Turco SJ, Leiby DA, Toro L, et al. Carbohydrate and LPG expression in Leishmania Viannia subgenus. J Parasitol. 1997; 83(4): 671-8.

Myskova J, Dostálová A, Penicková L, Halada P, Bates PA, Volf P. Characterization of a midgut mucin-like glycoconjugate of $\mathrm{Lu}$ tzomyia longipalpis with a potential role in Leishmania attachment. Parasit Vectors. 2016; 9: 413.

Pimenta PF, Turco SJ, McConville MJ, Lawyer PG, PerkinsPV, Sacks DL. Stage-specific adhesion of Leishmania promastigotes to the sandfly midgut. Science. 1992; 256(5065): 1812-5.

Pimenta PFP, Saraiva EM, Rowton E, Modi GB, Garraway LA, Beverley SM, et al. Evidence that the vectorial competence of phlebotomine sand flies for different species of Leishmania is controlled by structural polymorphisms in the surface lipophosphoglycan. Proc Natl Acad Sci USA. 1994; 91(19): 9155-9.

Ready PD, Arias JR, Freitas RA. A pilot study to control Lutzomyia umbratilis (Diptera: Psychodidae), the major vector of Leishmania braziliensis guyanensis, in a peri-urban rainforest of Manaus, Amazonas state, Brazil. Mem Inst Oswaldo Cruz. 1985; 80(1): 27-36.

Scarpassa VM, Alencar RB. Lutzomyia umbratilis, the main vector of Leishmania guyanensis, represents a novel species complex? PLoS ONE. 2012; 7(5): e37341.

Soares RP, Altoé EC, Ennes-Vidal V, da Costa SM, Rangel EF, Souza $\mathrm{N}$, et al. In vitro inhibition of Leishmania attachment to sandfly midguts and LL- 5 cells by divalent metal chelators, anti-gp 63 and phosphoglycans. Protist. 2017; 168(3): 326-34.

Soares RP, Cardoso TL, Barron T, Araujo MS, Pimenta PF, Turco SJ. Leishmania braziliensis: a novel mechanism in the lipophosphoglycan regulation during metacyclogenesis. Int J Parasitol. 2005; 35(3): 245-53.

Soares RPP, Macedo ME, Ropert C, Gontijo NF, Almeida IC, Gazzinelli RT, et al. Leishmania chagasi: lipophosphoglycan characterization and binding to the midgut of the sandfly vector Lutzomyia longipalpis. Mol Biochem Parasitol. 2002; 121(2): 213-24.

Soares RPP, Margonari C, Secundino NFC, Macêdo ME, Costa SM, Rangel EF, et al. Differential midgut attachment of Leishmania (Viannia) braziliensis in the sand flies Lutzomyia (Nyssomyia) whitmani and Lutzomyia (Nyssomyia) intermedia. J Biomed Biotechnol. 2010; 2010: 439174.

Wilson R, Bates MD, Dostalova A, Jecna L, Dillon RJ, Volf P, et al. Stage-specific adhesion of Leishmania promastigotes to sand fly midguts assessed using an improved comparative binding assay. PLoS Negl Trop Dis. 2010; 4(9): e816.

Young DG, Duncan MA. Guide to the identification and geographical distribution of Lutzomyia sand flies in Mexico, the West Indies, Central and South America (Diptera: Psychodidae). Mem Am Entomol Inst. 1994; 54: 881 pp. 